\title{
PELVIC REPOSITIONING IN LOW BACK PAIN PATIENTS
}

\author{
DG Wilder $^{1}$, E Owens ${ }^{2}$, MR Gudavalli $^{3}$, RD Macken ${ }^{4}$, T Xia $^{3}$, R Vining $^{3}$, \\ K Pohlman ${ }^{3}$, L Corber ${ }^{3}$, W Meeker $^{3}$, C Goertz $^{3}$, J G. Pickar ${ }^{3}$ \\ 1. Biomedical Engineering Dept., University of Iowa, Iowa City, IA, USA \\ 2. Northwestern Health Sciences University, Bloomington, MN, USA \\ 3. Palmer Center for Chiropractic Research, Davenport, IA, USA \\ 4. College of Engineering, University of Iowa, Iowa City, IA, USA
}

\section{Introduction}

As mechanical shock and vibration environments evolve, it is important to understand their potential effect on human operators with complex biomechanical characteristics comprised of passive and active mechanical components of varying mass, damping, stiffness, and actuation characteristics. Because the lumbar spine can exhibit local, shortcolumn buckling, stability of the seated human depends on postural control of the pelvis and trunk. ${ }^{1-2}$ The ability to sense the position of body parts in space depends on higherorder integration of proprioceptive, vestibular and visual information. Mechanoreceptors in the skin, muscles and joints can all contribute to proprioception. ${ }^{3}$ Limb repositioning has long been used to study the mechanisms of proprioception. ${ }^{4}$ We have been evaluating the ability of seated low back pain patients to reposition their pelvis. The results have implications for isolation design and standards development.

\section{Methods}

Using electromagnetic position and orientation sensors attached to the skin over T1, L1, L3, and S1, 66 low back pain patients (41 women, 25 men; 91\% low back pain duration > 1 year) were evaluated for their ability to reposition their pelvis while seated, without a backrest, hands crossed on their chest, on a small platform that could rotate about a transverse axis approximately aligned with the centers of rotation of the patient's femoral heads. Each patient was instructed to locate a pelvic target position, tilt their pelvis either forward or backward for 3 cycles, and then return to the target positions.

\begin{tabular}{|c|c|c|}
\hline Test Abbreviation & Target Position & Movement Initiation \\
\hline NF $=$ & Neutral (self-selected) & Forward \\
\hline FF $=$ & Forward & Forward \\
\hline FB $=$ & Forward & Backward \\
\hline $\mathrm{BF}=$ & Backward & Forward \\
\hline $\mathrm{BB}=$ & Backward & Backward \\
\hline $\mathrm{NF}=$ & Neutral & Forward \\
\hline $\mathrm{NB}=$ & Neutral & Backward \\
\hline
\end{tabular}

Blindfolded, each patient accomplished the repositioning task using the six combinations of target position and direction of movement initiation listed in the Table. Accuracy of repositioning was assessed by changes in the length of the spine from S1 to T1 (mm), 
horizontal location of the T1, L1 and L3 sensors with respect to the sacrum (mm), and lumbar flexion angle (deg) were calculated.

\section{Results}

The mean error (difference between initial and final positions for each test) was normalized by the difference between the mean maximum and mean minimum movements for each outcome. This was averaged across all six pelvic repositioning tests (NF, FF, FB, BF, BB, NF, NB). No tests for significance differences have been conducted. Measurements on 66 individuals indicates that the standard deviation exceeds the mean of the error.

\begin{tabular}{|c|c|}
\hline Normalized errors & $\begin{array}{c}\text { Error/(Max-Min) } \\
\text { Average across movements }\end{array}$ \\
\hline Length of Spine error S1 to T1 & 0.070 \\
\hline T1 horizontal position error with respect to Sacrum & 0.076 \\
\hline L1 horizontal position error with respect to Sacrum & -0.006 \\
\hline L3 horizontal position error with respect to Sacrum & -0.047 \\
\hline Lumbar Flexion Angle error & 0.046 \\
\hline
\end{tabular}

\section{Discussion}

In this pilot study, a method was explored for evaluating the ability of low back pain patients to reposition their pelvis. Sixty-six of a planned sample size of 200 patients have been evaluated before treatment for low back pain. Although not evaluated for statistical significance, the normalized errors of motions were less than 8\%, with the position of L1 with respect to the sacrum having the lowest normalized error. Insight developed from work like this will help determine the importance of considering control of posture in static or vibrating, seated conditions. There are several limitations of the described dataset: it represents only individuals with low back pain, data are still being collected, and only descriptive statistics have been evaluated.

Acknowledgements Under the leadership of Dr. Joel Pickar, this project entitled: Effect of Spinal Manipulation on Sensorimotor Functions in Low Back Pain Patients, was supported by Grant Number U19 AT004137 from the National Center for Complementary and Alternative Medicine (NCCAM). This investigation was conducted in a facility constructed with support from Research Facilities Improvement Program Grant Number C06 RR15433-01 from the National Center for Research Resources, NIH.

\section{References}

1. Wilder DG, Pope MH, Frymoyer JW (1988) The biomechanics of lumbar disc herniation and the effect of overload and instability. American Back Society Research Award. J Spinal Disorders 1(1):16-32

2. Wilder DG, Aleksiev AR, Magnusson M, Pope MH, Spratt K, Goel V (1996) Muscular response to sudden load: A tool to evaluate fatigue and rehabilitation. Spine 21(22):2628-2639

3. Gandevia, S.C. Kinethesia: roles for afferent signals and motor commands. In: Handbook of Physiology. Section 12: Exercise: Regulation and Integration of Multiple Systems. Rowell LB and Shephard JT. Bethesda, MD: American Physiological Society. (4):128-172, 1996.

4. Goodwin GM (1976) The sense of limb position and movement. Exerc.Sport Sci.Rev. 4(1):87-124 